\title{
MIGUEL DE QUINTANA, MAESTRO CANTERO, ARQUITECTO Y ESCULTOR EN LA SEVILLA DEL SIGLO XVIII
}

\section{MIGUEL DE QUINTANA, MASTER STONEMASON, ARCHITECT AND SCULPTOR IN SEVILLE OF THE XVIII CENTURY}

\author{
Teodoro Falcón Márquez \\ Real Academia de San Dionisio de Jerez de la Frontera. España \\ ORCID: 0000-0002-1300-708X \\ tfm@us.es
}

Miguel de Quintana fue un cantero de Cantabria que se estableció en Sevilla en el primer tercio del siglo XVIII. Aquí llegó a ser maestro cantero de la catedral, y trabajó con Leonardo y Matías de Figueroa en los más importantes edificios barrocos de la ciudad, especialmente en el antiguo convento de la Merced (Museo de Bellas Artes), en el Colegio Seminario de San Telmo y en la iglesia de Santa Catalina. Su obra más importante es la portada del actual Museo de Bellas Artes de Sevilla.

Palabras clave: Arquitectura barroca sevillana; Miguel de Quintana; Leonardo de Figueroa; Matías de Figueroa.

Miguel de Quintana was a stonemason from Cantabria, established in Seville in the first third of the XVIII century, where he became a master stonemason of the cathedral. I work with Leonardo and Matias de Figueroa in the most important baroque buildings of the city, especially in the old convent of La Merced (Museum of Fine Arts), in the sacramental chapel of Santa Catalina. His most important work is the cover of the current Museum of Fine Arts of Seville.

Keywords: Sevillian baroque architecture.; Miguel de Quintana; Leonardo de Figueroa; Matias de Figueroa.

Los canteros formaron parte de una larga serie de artífices de la piedra, que procedentes del norte de España, se establecieron en el antiguo Reino de Sevilla desde el siglo XV al XVIII, en donde trabajaron principalmente en su diócesis, así como para reales edificios y diversas casas aristocráticas. Miguel de Quintana, a quien se dedica por primera vez un artículo, estuvo activo en el primer 
tercio del siglo XVIII. Colaboró y mantuvo estrecha amistad con otros canteros de la misma procedencia, tales como: Ángel Gómez Bayas, natural de Setién, de la Trasmiera (Cantabria), antiguo arzobispado de Burgos. Con los Blanco, una saga que procedía del lugar de Soto, concejo de Ribadesella (Asturias), aunque el principal miembro de esta familia, Juan Antonio Blanco, nació en Sevi1la. Lorenzo Fernández de Iglesias, que era natural de Quintana del Monegro (Cantabria), jurisdicción de la villa de Reinosa, diócesis de Burgos. Francisco de Ganzarain (Gainzaráin), vizcaíno, yerno de Lorenzo Fernández de Iglesias. Francisco Gómez Septier (Setién), natural de Setién, en la Trasmiera. De Cantabria procedía la saga de los Jordán, que se inicia con Francisco y prosigue con Silvestre y sus hijos: Fernando, Manuel y José. A ellos hay que sumar a Andrés Zabala, natural de Bolívar (Vizcaya), entre otros. Posiblemente debió ser de ascendencia vasca, Vicente Bengochea, uno de los canteros de la portada del palacio de San Telmo de Sevilla, quien llegó a ser arquitecto de la Real Fábrica de Tabacos de esta ciudad ${ }^{1}$.

Miguel de Quintana es un descubrimiento de la segunda mitad del siglo XX y comienzos del XXI. Las primeras noticias documentales sobre él las facilitó Antonio Sancho, indicando que este maestro cantero trabajaba en 1724 en la portada principal del Palacio de San Telmo, año en el que estaba también al servicio de Leonardo de Figueroa en la iglesia de Santa Catalina. En este templo intervino en la capilla Sacramental, ejecutando el zócalo de piedras rojas y negras, así como la imagen de la Fe que corona su linterna ${ }^{2}$. Mercedes Jos aportó que Quintana ejecutó en la sacristía de la capilla de San Telmo el aguamanil y una mesa de mármol rojo ${ }^{3}$. Por su parte Ana Mendioroz documentó que fue el artífice de la portada principal del convento de la Merced (Museo de Bellas Artes), y que en 1726 arrendó una casa del Cabildo catedral en el Postigo del Aceite $^{4}$. Francisco Herrera facilitó algunos datos biográficos sobre su origen: matrimonio en Sevilla en 1706, así como el arriendo de dos viviendas en la feligresía del Sagrario ${ }^{5}$. También se ha publicado una monografía y varios artículos sobre el antiguo Colegio Seminario de San Telmo, en los que se documenta

${ }^{1}$ Algunas noticias sobre estos canteros, que ampliaremos con posterioridad, en: Sojo y Lomba, 1933: 66-67. Sancho Corbacho, 1952: 68-85. Garmendia Arrubarrena, 1986. Herrera García,1988: 9-26 y nota 6. Alonso Ruiz, 1991. Falcón, 1991b: 256-262. Díaz Fernández, 2003: 507-521. Cajigas Aberasturi, 2019. Sancho Corbacho denomina a Bengoechea como Catalán Bengoechea, aunque no figura así en ningún documento. Por otra parte no aparece como tal en el Diccionario de canteros vizcaínos, de Barrio Loza/Moya Valgón, 1981: 173.

2 Sancho Corbacho, 1952: 78 y 95.

3 Jos López, 1986: 25.

4 Mendioroz Lacambra, 1989: 261-266; Mendioroz, 1993: 151-154, notas 48 y 49.

${ }_{5}^{5}$ Herrera García, 1990: 120, nota 44. 
la intervención de este maestro cantero durante las décadas de 1720-30, bajo las directrices de Leonardo y Matías de Figueroa, con la frecuente extracción de piedras con destino a este edificio, desde las canteras de Mijas (Málaga), Morón (Sevilla) y el Puerto de Santa María (Cádiz), así como su actividad como escultor ${ }^{6}$. Finalmente, Fernando Quiles indica que el Cabildo catedral le concedió licencia en 1724 para pasar a una obra de cantería en el convento de Ntra. Sra. de la Merced de esta ciudad ${ }^{7}$. Hasta el momento su actividad en Sevilla se halla documentada a partir de 1706, cuando se casa en esta ciudad, hasta 1733 cuando percibe cierta cantidad por el Cabildo catedral, por la realización de unas ventanas en la Contaduría del templo metropolitano.

\section{DATOS BIOGRÁFICOS E INICIOS PROFESIONALES: LA ETAPA OSCURA (1706-1722)}

Quintana era natural de Cabárceno (Cantabria), en la Trasmiera, lugar que entonces pertenecía a la diócesis de Burgos. Este dato consta en varios documentos. En primer lugar, en su partida de casamiento, con María Gómez, en la iglesia del Sagrario de Sevilla. La ceremonia tuvo lugar en 20 de junio de $1706^{8}$. El matrimonio se estableció en la feligresía del Sagrario, cambiando con frecuencia de domicilio, a causa de las mejoras de su nivel de vida y, sobre todo, al hecho de llegar a tener una familia numerosa, así como al albergar a familiares y personal de servicio.

Consta que a partir de 1715 residía en esa collación. En 25 de mayo Rafael de Nava, en nombre del convento de Madre de Dios, arrendó a Quintana una casa en la feligresía de Santa María, por tiempo de dos años, por valor de 21 reales al mes. Figura como fiador el maestro cantero Ángel de Bayas Gómez. Al

${ }^{6}$ Falcón, 1991a; 1991b: 256-262; 1992; 2014: 277-290.

${ }^{7}$ Quiles, 2007: 331-332, 336.

${ }^{8}$ Institución Colombina. Archivo parroquial del Sagrario (APSa). Matrimonios. Libro $n^{\circ} 22$, f. 28r. (al margen: Miguel de Quintana con María Gómez): "En veinte de junio de mil y setecientos y seis años, yo el Dr. D. Pedro Márques Yoya, teniente de cura del Sagrario de la Santa Yglesia Metropolitana de Sevilla, con licençia del Sr. Dr. Alonso Garçia Valladares, cura más antiguo del dicho Sagrario, con mandamiento del señor juez de la iglesia y aviendo anteçedido los requisitos del derecho y las amonestaciones que disponiendo el Santo Conçilio de Trento, y no abiéndole resultado canónico impedimiento, desposé por palabras de presente que hicieron verdadero y lexítimo matrimonio a Miguel de Quintana, natural del lugar de Cavarsano, en las montañas, arçovispado de Burgos, hijo de Simón de Quintana y de Juliana Garçía, juntamente con María Gómes, natural desta ciudad, hija de Françisco Gómes y de Cipriana García....siendo testigos Salvador Coronil, Silbestre Jordán, Ángel de Bayas Gómez y otros....". El documento había sido trascrito parcialmente por: Herrera García, 1990: 174, nota 44. En otro documento fechado en 7 de octubre de 1726, indica que era natural del Arzobispado de Burgos. Mendioroz, 1993: 151. 
año siguiente, en 8 de mayo de 1716, recibía en arrendamiento una casa del Cabildo catedral, por tiempo de dos años, por valor de dos ducados al mes. Bayas consta de nuevo como fiador ${ }^{9}$. En la década siguiente se estableció en el Postigo del Aceite (Rastrillo del Aceite) n ${ }^{\circ}$ 5, "mano izquierda", actual c/Almirantazgo, en donde vivía en $1729^{10}$. En 1731 se trasladó a la "Resolana, mano derecha", no 485. Vivían en esta casa once personas ${ }^{11}$. La vivienda se hallaba en la "Resolana de la Caridad", actual calle Temprado. Estos documentos y otros avalan la estrecha amistad que Quintana tuvo con diversos canteros establecidos en Sevilla en el primer tercio del siglo XVIII. Entre ellos figuran: Ángel de Bayas Gómez, Silvestre Jordán, Francisco Ganzaráin, Juan Antonio Blanco, Tomás Alonso Moreno, Francisco Bermúdez y Juan Chirino ${ }^{12}$. En los documentos se alude a la interrelación que tuvieron en calidad de testigos de sus correspondientes bodas, bautizos y testamentos, en el alquiler de viviendas, así como actuando de fiadores en diversos contratos profesionales ${ }^{13}$. En sus primeros años de trabajo en esta ciudad, a Quintana se le denomina indistintamente maestro cantero (1715) y "peón de la Santa Iglesia" (1716) ${ }^{14}$.

Trabajó en sus inicios a las órdenes de Lorenzo Fernández Iglesias (16551721), maestro cantero de la catedral ${ }^{15}$, quien en 1703 reconocía la bóveda de la capilla de la Virgen de la Antigua, afectada por los efectos de un terremoto. En 1708 presentaba una Memoria alusiva a las obras que necesitaba el templo, y en 1712 informaba de las obras de cantería que había llevado a cabo en las cubiertas y remates de este edificio ${ }^{16}$. En un informe sobre la iglesia de Santa María de Arcos, fechado en 1696, se titula maestro mayor de obras de cantería de esta ciudad. Consta en las Nóminas y Salarios de la catedral como maestro cantero

${ }^{9}$ Herrera García, 1990: 120.

10 APSa. Padrones n 20 (1725-1727): "Padrón Quarto del Sagrario. Año de 1725", s/f. Figuran: Miguel de Quintana, María de la Concepción, Ángel de Quintana, Luisa de Quintana y Carlos Corral. En el Padrón de 1726 constan además: María Gómez, Juan de Cierra (Sierra) y Manuel de Sebillos. Asimismo, indica que vivía en esa casa en el contrato para realizar la portada de la iglesia de la Merced en 1729.

${ }^{11}$ APSa. Padrones n ${ }^{\circ} 23$ (1731-1732), f. 45r: Miguel de Quintana, María Gómez, Luisa de Quintana, María de Quintana, Isabel de Quintana, Ángel de Quintana, Juan Delgado, Juan de Aranda, María Gregoria, Ignacio Juan Méndez y Bárbara de Urrutia.

12 Todos ellos figuran en las nóminas de la construcción del antiguo retablo mayor de la iglesia del Sagrario, dirigidos por Jerónimo Balbás y Duque Cornejo. No consta Quintana porque era un peón. Romero Torres, 2014: 259-276.

${ }_{13}$ El cantero Ángel Baya puso a su hijo el nombre de Miguel, sin duda por su relación con Quintana. APSa. Libro de Bautismos $\mathrm{n}^{\circ}$ 53, f. 179v.

${ }_{14}$ Herrera García, 1990: 120.

15 Sancho Corbacho, 1934: 21-28, 1952: 44, 122-126, 179-180. Herrera García, 1988 : 9-27; 1990: 48-53.

${ }^{16}$ Falcón, 1980: 62-64 y 168-171. 
desde $1707^{17}$. Intervino en numerosas iglesias diocesanas, tales como la del Sagrario de la catedral y en templos de Arcos y de Jerez ${ }^{18}$, entre otros. Quintana siendo oficial de cantero, acompañó en 1718 a Fernández de Iglesias a Cañete la Real. En 1713 Lorenzo Fernández mostró una planta realizada por él de la iglesia de San Pedro de Arcos, para ampliarla por los pies. En esta obra participó su yerno Francisco de Gandarain (Gainzaráin). A partir de 1722 Quintana ejerce como maestro cantero de la catedral, encargándose de la provisión de piedras para el solado del trascoro. Por ello fue agasajado por el Cabildo, "en atención a su mucha habilidad". Finalmente, en 1724 pidió licencia para ausentarse por un mes en el trabajo de este templo, ya que le ofrecían 14 reales por participar en unas obras del convento de la Merced, en lugar de 5 reales que percibía en el templo metropolitano. Por esa razón no volvió a estar en su nómina ${ }^{19}$. Pese a ello siguió colaborando de forma puntual en obras de reparo de la catedral. Consta que en 1733 sacó y condujo hasta este templo una serie de carretadas de piedra martelilla de Jerez para las ventanas de la Contaduría ${ }^{20}$. Se trata de la última obra documentada.

Sin duda la labor más notable de Fernández de Iglesias fue la ejecución de la portada principal del palacio arzobispal de Sevilla, que acometió entre 17031705. Previamente, a partir de 1699 se había iniciado la construcción del zócalo de piedra de las fachadas, bajo la dirección del maestro mayor Pedro Romero. El 14 de septiembre de 1699 los maestros canteros Francisco Gómez Septier (Setién) y Antonio Gil Gataón, se obligaron ante el escribano Pedro Prieto, a sacar y desbaratar de las canteras de Carmona 500 carretadas de piedra para la obra "que se ha de hacer en el Palacio Arzobispal", a razón de 28 reales cada carretada. En el documento se estipula que las carretadas se debían entregar en el plazo de cuatro meses, contabilizados desde el 15 de septiembre de 1699, hasta el 14 de enero de $1700^{21}$. Las portadas de este palacio se iniciaron en 1703. En 24 de noviembre de ese año Lorenzo Fernández de Iglesias suscribía una escritura para traer de las

17 Archivo Catedral de Sevilla (ACSe). Sección IV. Libro 316 (09656), s/f. En 1712 consta en la nómina de 1 de junio, que realizó el remate de la Puerta Grande (Asunción). Debemos advertir que en estas Nóminas y Salarios no suelen aparecer los nombres de los peones.

18 Falcón, 1977; 1983.

19 Quiles, 2007: 331-336.

${ }^{20}$ ACSe. Sección IV. Libro de Salarios 04821 (802). Al margen (Piedra martelilla): "En 24 de Diziembre de 1733 se libraron a Miguel de Quintana, maestro cantero, 600 rs. de vn. por la saca y conduzión de cinco carretadas de piedra martelilla de Jerez, para reparo de las obras de esta Santa Iglesia” (Ventanas de la Contaduría).

${ }^{21}$ Archivo Municipal de Sevilla (AMSe), libro 158 de Actas Capitulares, $2^{\mathrm{a}}$. Cabildo de 9 de noviembre, s/f. Sancho Corbacho, 1934: 37, nota 1. Archivo Histórico Provincial. Sección de Protocolos Notariales. Leg. 17.109. Falcón, 1997: 75-85. Falcón, 2018: 66-76. Falcón, 2011: 225-251. 
canteras de Jerez 700 varas de piedra de las canteras de Martelilla y 140 varas de mármol de las canteras de Mijas. Contratos complementarios se suscribieron en 19 de mayo y 21 de octubre de 1704, así como en 13 de enero de 1705. También participaron los canteros Silvestre Jordán y Juan Antonio Blanco, quien remató la portada principal en $1705^{22}$. Silvestre Jordán había participado en las obras de cantería de la capilla sacramental de San Lorenzo y en la iglesia de la O, desde 1697 hasta 1702 junto a Antonio Gil Gataón ${ }^{23}$. Vivía en la feligresía del Sagrario, en la Puerta del Arenal24. Falleció en 1707, prosiguiendo su labor sus hijos, canteros de la catedral, que intervinieron además en varios templos diocesanos ${ }^{25}$. Aunque no hay documentación que lo avale, debemos suponer que Quintana participó en esta portada como cantero. Por un lado, porque, como hemos dicho, estaba entonces al servicio de Fernández de Iglesias. Por otra parte, hay que tener en cuenta que el cantero Silvestre Jordán, quien asimismo consta extrayendo piedras para esta portada, intervino como testigo en la boda de Quintana en 1706, poco después de haberse concluida su construcción, lo que evidencia no solo vínculos de amistad, sino también profesionales.

\section{DE CANTERO A MAESTRO CANTERO, ARQUITECTO Y ESCULTOR. SU CONSAGRACIÓN CON LOS FIGUEROA}

A lo largo de las décadas de 1720 y 1730 tuvo lugar el salto cualitativo de este protagonista, que se consagró como maestro cantero y arquitecto, en un equipo integrado por Leonardo y Matías de Figueroa, más el pintor Domingo Martínez y el escultor Pedro Duque Cornejo. Asimismo, actuó como escultor, en una faceta menos conocida, pero importante, por lo que debería ser más reconocida su labor. Solo un reducido número de obras merecen otorgarle un mayor prestigio. Como veremos, en el antiguo Colegio Seminario de San Telmo realizó en 1723 los relieves de los bustos de Santo Domingo y San Telmo, que se hallan en el patio principal, en el arco de ingreso a la capilla. Dos años después (1725), aunque no están documentadas, le atribuimos las esculturas y relieves que decoran la portada del vestíbulo que da acceso al citado patio. Las tallas representan a virtudes, de la que es reconocible la Fortaleza, que porta la

${ }^{22}$ Falcón, 2018: 73-75. Con destino a esta portada es, sin duda, el documento siguiente: "Luis de Castro, patrón de barco, vecino de Coria y residente en Sevilla, declara el 21 de octubre de 1704, que está obligado con Silvestre Jordán, maestro cantero, a transportar 33 carretadas de mármol blanco desde Fuengirola (Mijas), hasta Sevilla, dejándolas al pie de la Torre del Oro". Herrera García, 1990: 98.

${ }_{23}$ Sancho Corbacho, 1934: 37-38 y 116. Falcón, 2011: 225-251.

${ }^{24}$ APSa Padrones. Libro ${ }^{\circ} 13$ (1704-1706), no 380 . Vivía con su esposa Josefa Victoria y con sus hijos José, Fernando, Manuel y María.

${ }^{25}$ Herrera García, 1990: 93-98 y 173. Falcón, 2011: 225-251. 
columna. En 1729, según documentó Sancho Corbacho, talló la imagen de la Fe que corona la Capilla sacramental de la iglesia de Santa Catalina. Pero si todo esto fuera poco es el arquitecto y escultor que realizó entre 1729-30 la labor arquitectónica y escultórica de la portada de la iglesia del antiguo convento de la Merced, hoy portada principal del Museo de Bellas Artes de esta ciudad, como publicó Mendioroz.

En esta etapa Miguel de Quintana pasó de ser cantero, a maestro cantero de la catedral en 1722, como consta por acuerdo del cabildo de 31 de agosto. Ello acarreó el incremento de su salario. En mayo de 1724 solicitó al Cabildo licencia "para pasar a una obra de cantería en el convento de Ntra. Sra. de la Merced, en atención a no tener cosa precisa y estar muy pobre, pues no le alcanzaban los 5 reales que tiene de salario para su mucha familia y en esta obra que será de un mes, y en que gana cada día 14 reales, se podrá desahogar". La licencia se le concedió en 19 de mayo de ese año. Aunque aplazó su regreso dos meses, lo cierto es que no se reintegró ${ }^{26}$. Quintana no fue del todo claro al solicitar esa licencia, ya que desde el año anterior (1723) trabajaba al servicio de Leonardo de Figueroa en el Colegio Seminario de San Telmo, por lo que el bajo salario que percibía de la catedral y el tiempo que le dedicaba, eran un lastre para él. La petición de licencia le sirvió de excusa para intervenir en el edificio del convento de la Merced a las órdenes del mismo arquitecto, al que se añadirá en 1724 un tercero, la capilla sacramental de Santa Catalina. Dado que la intervención de Quintana en todos ellos fue simultánea, lo abordaremos por separado.

\section{CONVENTO DE LA MERCED}

Su participación en este cenobio de mercedarios descalzos se llevó a cabo en dos etapas. La primera entre 1724-25, en la reconstrucción parcial del claustro grande y la segunda, entre 1729-30, proyectando y ejecutando la portada que inicialmente se colocó a los pies de la iglesia, en la c/Bailén (del ABC), la cual se trasladó para servir de acceso al Museo de Bellas Artes, en el curso de la nueva fachada hacia la plaza, que proyectaron y dirigieron Alberto Balbontin de Orta y Antonio Delgado Roig entre 1942-45. La decoración de esta nueva fachada se inspira en la de la segunda planta del Claustro Grande, renovada por Leonardo, a base de pilastras avitoladas de orden jónico y ventanas rematadas con molduras de perfil quebrado, con orejetas. El claustro principal amenazaba ruina en 1724, por lo que en 6 de marzo de ese año Leonardo de Figueroa, maestro arquitecto; Alonso López, maestro de albañilería y Juan

${ }^{26}$ Quiles, 2007: 331-336.

LABORATORIO DE ARTE 33 (2021), pp. 201-224, ISSN 1130-5762 e-ISSN 2253-8305 - DOI http://dx.doi.org/10.12795/LA.2021.i33.11 
Tomás Díaz, maestro de carpintería ${ }^{27}$, presentaron unas trazas y una memoria de obras ante escribano, en las que especifican las tareas a emprender para su rehabilitación. En la planta baja, las columnas pareadas de mármol blanco proyectadas por Juan de Oviedo en 1602 se remataron con 22 cimacios "de piedra fina", se rehicieron los arcos, reforzados con tirantes de hierro y se renovaron los alfarjes. La galería alta se hizo de nuevo. Es cerrada, en la que se colocaron 22 balcones, cuyos vanos se flanquearon por dobles pilastras avitoladas y rematados por molduras con orejetas. Asimismo, se hicieron de nuevo las cubiertas. El presupuesto de la obra se estipuló en 9.113 escudos de plata. Recordemos que para intervenir en las tareas de derribo y reconstrucción del claustro fue solicitado Miguel de Quintana, a quien dio licencia el Cabildo catedral en 19 de mayo. Un nuevo reconocimiento de las obras por parte del equipo facultativo tuvo lugar el 3 de junio del mismo año de $1724^{28}$. Quintana, siendo maestro cantero del Colegio Seminario de San Telmo, intervino ese verano en las tareas de derribo en el claustro del convento, cuyas carretadas de piedra se utilizaron para los cimientos del claustro y fachada principal del Real Colegio que se estaba construyendo ${ }^{29}$. A partir de entonces se inició la construcción del banco de la fachada, con carretadas de piedra de las canteras de Morón y de Mijas, dirigidas por Quintana y supervisadas por Matías de Figueroa "maestro de la obra de este Colegio" 30

Sin duda la obra que ha consagrado a Quintana ha sido la ejecución de la portada de la iglesia de este convento, que ahora sirve de acceso al Museo de Bellas Artes. Su contrato se formalizó el 10 de marzo de $1729^{31}$. En él se titula arquitecto y maestro cantero, vecino del Rastrillo del Aceite. Intervienen como fiadores los oficiales Tomás Alonso Moreno, Francisco Bermúdez y Juan Chirino. En las condiciones para su ejecución se especifica que ha de ser de mármol y piedra martelilla, y se alude a "un diseño o modelo que se ha demostrado y está sombreado y firmado por el reverendo padre Francisco Bartolomé de Rojas, comendador de dicho convento, y de mí el dicho principal" (Figura 1). Entre las modificaciones que propone Quintana, especifica sustituir las columnas

${ }^{27}$ Este maestro carpintero trabajó asiduamente en el equipo de Leonardo de Figueroa, principalmente en San Telmo, en donde realizó varios retablos de la capilla: Jos López, 1986. Falcón, 1991a.

${ }_{28}$ Sancho Corbacho, 1952: 98, nota 129. Mendioroz, 1993: 74-75, nota 34.

${ }_{29}$ Archivo Histórico de la Universidad de Sevilla (AHUSe). Fondo Histórico de San Telmo. Libro 22, pp. 365-367: "It. 720 rs. de plata que se pagaron al R.P. Fr. Bartolomé de Rojas de Ntra. Sra. de la Merced, casa grande de esta ciudad, en virtud de recado de data de 28 de julio de 1724, por 72 pesos escudos de plata que importó el valor de 244 carretadas de piedra, que del derribo del claustro de dicho convento se compraron para los cimientos del claustro y fachada que se está ejecutando en este Real Colegio". Libro 170, f. 57v.

${ }^{30}$ Falcón, 2014: 278-280.

31 Mendioroz, 1993: 151-153. 
salomónicas previstas por otras retalladas de orden compuesto, "que sean de bajorrelieves, por la falta de espacio". Lo construido fueron columnas pareadas sobre plintos, con una decoración a base de guirnaldas y frutos, y en el tercio inferior se puso el blasón de la Orden de la Merced. Las medidas de este cuerpo debían ser de "10 varas y cuarto" (cerca de 8,50 m.). Sobre la rosca del arco ejecutó una rica decoración a base de cortezas y motivos vegetales, entre los que figuran los prótomos de dos niños-genios alados, con extremidades bífidas a base de cola de tritón y vegetal. Corona este trasdós del arco el anagrama mariano, con corona real, y una abigarrada ornamentación a base de conchas, roleos y frutos.

Respecto al segundo cuerpo afirma que el primer diseño "está desproporcionado, según buena simetría y proporción”, por lo que habrá de ejecutarse como figura en el nuevo. Se hizo de columnas salomónicas sin decorar, de orden compuesto (Figura 2). A eje con las columnas extremas del cuerpo bajo, se colocaron jarrones pétreos. Asimismo, se indica entre otras condiciones: "como también lo han de ser una imagen de Nuestro Señora y dos captivos a los lados" (Figura 3). En realidad lo que talló Quintana, fue la imagen titular sobre una nube de querubines, y las que representan a San Pedro Nolasco, el fundador de la Orden, y al rey Jaime I de Aragón. La estética de estas tallas muestra cierto paralelismo con la obra de Pedro Duque Cornejo. Recientemente García Luque ha propuesto que estas imágenes pudo realizarlas el escultor veneciano Domingo Grazelli (Domenico Grasselli), establecido en esta ciudad en 1713, cuando tenía unos 34 años. Aquí se casó y trabajó en 1720 a las órdenes de Diego Antonio Díaz en la decoración de la portada de San Miguel de Jerez. Con posterioridad habiendo entablado amistad con Quintana, en 1726 éste y su esposa apadrinaron a su hija María Micaela en su bautizo en la iglesia de Santiago. La atribución de la portada del convento al artista italiano la basa García Luque a raíz de la documentación que aporté referente a que Grazelli perteneció al equipo de Matías de Figueroa, realizando en 1734 las tallas de los evangelistas en las torres del patio de San Telmo ${ }^{32}$. Sin embargo, en el contrato de esta portada especifica que toda la talla la debe ejecutar él. Culmina el conjunto un frontón roto en cuyo centro hay un gran blasón de la Orden, con basamento decorado con motivos auriculares. Finalmente propone Quintana que, a la hora de ejecutar los cimientos de la portada, el convento nombre a "un maestro de la satisfacción, para que registre la seguridad de dicho cimiento". El valor estipulado de la obra ascendió a 16.000 reales de vellón, pagaderos en varias fases. La última, por valor de 1000 reales "cuando la obra esté acabada a satisfacción del maestro que fuere nombrado".

${ }^{32}$ Falcón, 1991: 121; Gaeta/García Luque, 2019: 389-396. 
Comentando lo que afirma Quintana en esas condiciones, parece que hubo un primer proyecto de la portada, presentado por el comendador Rojas, que el maestro cantero rectificó por no tener las proporciones adecuadas, haciendo algunos cambios en él. La obra definitiva muestra un rico repertorio ornamental, con relieve pictórico, que evidencia que los canteros que la labraron debieron tener delante una muestra gráfica. En otras publicaciones hemos documentado que en este tipo de obras solía haber un equipo integrado por un pintor, un maestro cantero, un escultor y un arquitecto, que velaba por la cimentación y anclaje de la portada. En esta obra el maestro cantero y escultor es Miguel de Quintana. El pintor es, sin duda, Domingo Martínez (h. 1688-1749), asiduo en el equipo de Leonardo y Matías de Figueroa. Diseñó la portada principal de San Telmo, así como unas lápidas conmemorativas en ese edificio, que labró Quintana. Martínez en 1727 había sido contratado junto a Miguel Moreno, para ejecutar las pinturas al temple que decoran el interior de la iglesia de este convento. En su producción artística muestra los conocimientos que tenía de los grandes tratadistas, cuyas obras figuraban en su legado testamentario. Respecto al modelo de columnas del cuerpo bajo de esta portada, con decoración de guirnaldas y frutos, lo repetirá en el proyecto de retablo de la Virgen de la Antigua de la catedral de Sevilla ${ }^{33}$. Por otra parte, esta portada ofrece paralelismos con la del Palacio arzobispal (1703-1705). En particular en la decoración, a base de niños alados sobre la rosca del arco, querubines, cortezas y motivos auriculares, además del tipo de columnas retalladas, con ornamentación a base de guirnaldas y frutos. Probablemente la coincidencia en ambas portadas se encuentra en el hecho de haber tomado referencias de láminas de los mismos libros; especialmente de Androuet de Cerceau, Album de Arquitectura (h. 1570-80), del que se conserva un ejemplar en el Colegio Oficial de Arquitectos de Madrid, procedente de la Compañía de Jesús de Sevilla, y del tratado de Arquitectura de Wendel Dietterlin $(1598)^{34}$.

Respecto al arquitecto "de la satisfacción" por parte del maestro cantero y de la comunidad, que debía supervisar la cimentación y estabilidad de la portada, no cabe duda que se trata de Leonardo de Figueroa (1654-1730). Fue el artífice de las reformas del claustro grande, quien había contratado a Quintana como maestro cantero para esta labor, y bajo cuyas órdenes se hallaba nuestro protagonista trabajando en San Telmo. Consta en 16 de diciembre de 1729, que Matías de Figueroa actuaba como fiador de su padre en esta obra $^{35}$. Según el primer contrato las obras deberían finalizar a fines de febrero del año siguiente. A causa de que Leonardo falleció el 10 de abril de 1730, y el hecho de que tres días antes había suscrito su último testamento, en el que expresaba hallarse imposibilitado, permite

\footnotetext{
33 Pleguezuelo, 2004: 210-211.

${ }^{34}$ Falcón, 2008/9: 117-134; 2018; 97-99.

${ }^{35}$ Mendioroz, 1993: 76.
} 
inferir que las tareas finales de esta portada debieron ser supervisadas por Matías José de Figueroa (1698-1767), como hizo con frecuencia en la dirección de las obras del Colegio de San Telmo.

\section{REAL COLEGIO SEMINARIO DE SAN TELMO}

Es uno de los edificios más monumentales de la ciudad, del que se conserva una abundantísima documentación, actualmente depositada en el Archivo Histórico de la Universidad de Sevilla (AHUSe). El antiguo Real Colegio fue fundado en 1681 por Real Cédula de Carlos II de 17 de junio. Su primera fase constructiva tuvo lugar desde 1682 a 1706, bajo la dirección del arquitecto Antonio Rodríguez. La segunda fase, en la que se hicieron las obras más notables, tuvo lugar desde 1721 a 1736 bajo la dirección de Leonardo y Matías de Figueroa. A este edificio le hemos dedicado una monografía y diversos artículos ${ }^{36}$. La intervención de Quintana en este edificio la hemos documentado entre 1723 y 1732, en donde trabajó a las órdenes de Leonardo y Matías de Figueroa. Sorprende que en la primera fecha figurara aún como cantero en las nóminas de la catedral, en un año -como veremos-en el que tuvo una gran actividad en el Colegio. A lo largo de diez años intervino en la extracción de piedras de diversas canteras, en su corte y pulimento para columnas y pilares; en el revestimiento de aplacados de mármoles y jaspes en los muros; en el banco y frontal del altar mayor; peldaños de escaleras; en la ejecución de fuentes, aguamanil y pilas de agua bendita; talla de bustos en relieve y ornamentación de lápidas conmemorativas, así como en la probable realización escultórica en una portada. Su campo de acción se circunscribió a la iglesia, claustro principal, enfermería, primitiva escalera principal, fachada y portada principal, entre otros lugares.

La nueva iglesia, proyectada e iniciada de cimientos en 1721, según trazas de Leonardo, se ubicó en el extremo oriental del eje que marca la puerta principal y el claustro. Aunque la obra de fábrica estaba concluida en octubre de 1723, su consagración tuvo lugar el domingo 23 de enero del año siguiente, siendo bendecida por el arzobispo don Luis Salcedo y Azcona. Los retablos de José Maestre, la decoración escultórica de Duque Cornejo, y la pictórica, a base de lienzos y pinturas murales de Domingo Martínez, se realizaron en años sucesivos. La participación de Quintana en este templo se inició a comienzos de 1723, percibiendo en 4 de febrero una importante cantidad por el sotabanco del retablo mayor, además de 177 reales por la pila del patio que está junto a la

${ }^{36}$ Falcón 1991a; 1991b: 256-262; 1992; 2014: 277-290. 
sacristía ${ }^{37}$. En abril ejecutó el basamento de jaspe para el púlpito de hierro forjado, que realizó José Rodríguez ${ }^{38}$. En julio, la pila de agua bendita de la sacristía $^{39}$. Dos años después percibía en 4 de junio de 1725 cierta cantidad por el frontal para el camarín de la iglesia, y en 15 de septiembre otra por el aguamanil de la sacristía, con piedras de jaspe rosado procedentes de Mijas, en el que figura una reproducción de la Torre del Oro. El conjunto se corona con un frontón de triple inflexión ${ }^{40}$.

Simultáneamente intervino en la construcción de la Enfermería, ubicada a la derecha de la iglesia, junto al ala oriental del Dormitorio de los Muchachos y próxima a la cocina. Se proyectó de planta cuadrada, con acceso al patio principal, y se organizaba en torno a un patio, con salas para los enfermos y habitación para el enfermero. Iniciada su construcción en septiembre de 1722, según diseño de Matías de Figueroa, Quintana intervino en abril del año siguiente, pagándosele cierta cantidad por las basas y cimacios para las columnas y por el brocal de pozo $^{41}$. En octubre ejecutó cuatro gradas de jaspe para la entrada, una pila con su taza y pedestal de mármol blanco para el patio chico, y una pileta de jaspe negro, con su cruz embutida, para poner "en la fuente pequeña que sale al claustro chico" ${ }^{42}$. El tándem Matías de Figueroa-Quintana sustituyó las columnas por pilares en abril de $1733^{43}$.

El patio principal es el centro neurálgico del edificio. De planta rectangular, presenta en la galería baja 7 por 5 arcos rebajados sobre pilares de sección rectangular. La planta alta es cerrada, con balcones. Este espacio responde al modelo de barroco polícromo creado por Leonardo, aunque su proyecto y ejecución se debe a Matías José de Figueroa (1698-1767). Sus obras comenzaron el 22

37 AHUSe. Libro 22, ff. 406-409. (Al margen: Retablos): "Item. 1267 rs. y 11 mrs. de plata corriente que de ella se pagaron a Miguel de Quintana maestro de cantería de este Real Colegio, en virtud de libranza de 4 de febrero del año pasado (1723) por 1911 rs. de vn., los 1723 y medio en que se ajustó el sotabanco de piedra de jaspe para el retablo grande que se está ejecutando para el altar mayor de la nueva iglesia, y los 177 rs. y medio restantes por un pedestal de mármol que hizo para la pila del patio que está junto a la sacristía de dicha iglesia".

38 AHUSe., libro 21, ff. 567-568. Falcón, 1991: 28, nota 31.

39 AHUSe., libro 21, ff. 574-575.

40 AHUSe., libro 23, ff. 459-460. "Se pagaron al citado maestro de cantería Miguel de Quintana en 15 de septiembre (1725) 30 pesos escudos de plata que importó el valor de dos carretadas y media de jaspe encarnado (de Mijas) que de su cuenta trajo a este Cabildo para labrar el aguamanil de la nueva sacristía de él, a razón de 10 pesos escudos la carretada y asimismo el costo de un poco de piedra negra que se compró para los embutidos de dicho aguamanil”. La ilustración del aguamanil en Falcón, 1991: 56.

${ }^{41}$ Falcón, 1991: 145-146. AHUSe., libro 21, ff. 567-569. Libro 69, f. 239.

${ }^{42}$ AHUSe., libro 21, ff. 579-580.

43 AHUSe., libro 21, ff. 475, 506 y 559. 
de septiembre de 1722, iniciándose por el frente oriental, delante de la iglesia ${ }^{44}$. Constan diversos pagos a Quintana por su intervención en este patio desde abril de 1723, cuando participó en la construcción de pilares ${ }^{45}$. Aquí hay que destacar la labor desarrollada por nuestro protagonista en calidad de maestro escultor. En las enjutas del arco central, que focaliza la entrada a la iglesia, labró en mármol blanco los bellísimos bustos en altorrelieve, que representan a Santo Domingo y San Telmo ${ }^{46}$ (Figura 4). En diciembre de ese año pintó y decoró estos relieves Domingo Martínez ${ }^{47}$.

En 31 de julio de 1733 Quintana recibió unos honorarios por tallar con inscripciones y relieves dos lápidas conmemorativas, de mármol con decoración dorada. Una se puso sobre el dintel de la puerta de la Enfermería. Había sido diseñada por Domingo Martínez ${ }^{48}$. En la parte superior figura el escudo real, flanqueado por las Columnas de Hércules, y en la inferior dos colegiales uniformados, que sostienen un mapa que representa la península de Florida y las Antillas. El texto es el siguiente:

"PARA LA HONRA Y GLORIA DE DIOS NVESTRO SEÑOR/Y BIEN DESTA REPVBLICA EL SR. D. CARLOS/II POR SU REAL DESPACHO DE 17 DE JUNIO DEL/AÑO DE 1681, CONCEDIÓ LA ERECCIÓN DES/ TE COLEGIO SEMINARIO, DONDE SE EDUCA/UNA DESVALIDA JUVENTUD, DEDICADA A LAS/ARTES DE LA NAVEGACIÓN Y FACULTADES MA/TEMÁTICAS, CUYA FUNDACIÓN SE DESEÓ DES/DE EL AÑO DE 1607. Y SE RESERVÓ POR LA/DIVINA PROVIDENCIA PARA EL TIEMPO DE SU/REYNADO, Y LA CONSTITUYÓ DEBAXO DEL/AMPARO DE SU REAL PATRONATO CON OTROS/MUCHOS PRIVILEGIOS.

44 En Junta de la Universidad de Mareantes celebrada en esa fecha consta:" Acordamos que se de principio a la referida obra de dicha enfermería y claustro, que ha de ser según la planta hecha por el maestro Matías de Figueroa, hijo de Leonardo de Figueroa, que había asistido a la obra principal de la iglesia, con entera satisfacción de esta Diputación". AHUSe, libro 21H, ff. 566, libro 169, ff. 239,252.

${ }^{45}$ AHUSe., libro 21, f. 568: "Item por el costo que tuvieron 14 piedras grandes que se labraron, bruñeron y pusieron por basas de los pilares del claustro que se está haciendo delante de la iglesia de este Colegio, en las cuales dichas basas están incluidas las dos piedras angulares, que todas entregó dicho maestro". libro 169, ff. 239, 252,266. Libro 23, f. 447.

${ }^{46}$ AHUSe., libro 169, f. 252:" Item por dos medios cuerpos de piedra mármol de Santo Domingo y San Telmo para poner en medio del ángulo del claustro en el arco de frente de la puerta de la iglesia, ajustados en 32 pesos escudos ambos (480 rs.)". Fotografías de estos relieves en: Falcón, 1991: 33 y 34.

${ }^{47}$ AHUSe., libro 21, f. 634: "Por el costo de un ramo de azucenas y dos diademas de hojalata para los dos santos que están en la fachada principal del claustro, 16 rs. y $17 \mathrm{mrs}$. Por el dorado de ellos y una estrella para Santo Domingo, 14 rs.".

48 AHUSe., libro 21, ff. 573 y 579, libro 169, f. 252. 
Y LA ENCOMENDÓ AL/CUIDADO DE LA UNIVERSIDAD DE MAREANTES/QUE SE COMPONE DE LOS DUEÑOS, CAPITANES, MA/ ESTRES Y PILOTOS QUE NAVEGAN LA CARRERA/DE INDIAS, CON CUYAS CONTRIBUCIONES SE/MANTIENE TAN PIADOSA OBRA QUE SE VE ADE/LANTADA CON ESTA NUEVA IGLESIA, CLAUS/TROS Y ENFERMERÍA POR EL ZELO Y APLICACI/ÓN DE LOS CABALLEROS DIPUTADOS QUE EXPRE/SA LA CORRESPONDIENTE LÁPIDA, EN CUYO/TIEMPO SE FIXA ÉSTA."

Asimismo, Quintana labró otra lápida de las mismas características, por la que percibió 24 pesos (360 reales). Se realizó para ser colocada en un ángulo del patio principal. Fechada en 1723, su texto alude al comienzo de las obras de la iglesia, claustro y enfermería en $1722^{49}$. En la parte superior figura el escudo real, flanqueado por el blasón de la Orden de Calatrava y por una nao. En la parte inferior dos niños alados sostienen una carta de navegación e instrumentos náuti$\cos ^{50}$. Su texto es el siguiente:

"D.O.M.S./REINANDO EN ESPAÑA EL S.D. PH. V SE COMENÇÓ ESTA YGLESIA, CLAVSTRO/Y ENFERMERÍA EL DÍA 9 DE ABRIL DEL AÑO DE 1722/SIENDO JUEZ PROTECTOR DE ESE R. COLEGIO SEMINARIO/EL S.D. MANVEL DE TORRES DEL CONSEJO DE S.M./EN EL RL. DE CASTILLA, REGENTE EN LA RL. AVDIENCIA/DESTA CIVDAD Y ALCAIDE ENGOBERNADOR DE LOS RL./ALCÁZARES DE ELLA Y MAYORDOMO Y DIPVTADOS/LOS CAPITANES D.MATEO PABLO DÍAZ/DE LAVANDRO I CÓRDOVA 24 PERPETVO DESTA CIVDAD/ALGVACIL M. DEL S. TRIBVNAL DE LA YNQUISICIÓN DESTE/ ARZOBISPADO,OBISPADOS DE CÁDIZ/Y CEVTA, DEL CONSEJO DE S.M. EN EL RL DE HAZIENDA/Y SV ADMINISTRADOR GENERAL DE LAS REALES ADVANAS I SALINAS DESTA CVDAD Y LAS DE SV JURISDICCIÓN D. MANUEL/SÁNCHEZ DVRÁN SECRETARIO DEL RL. FISCO DE/DICHO SANTO TRIBVNAL, I DON JVAN EVSEBIO GARCÍA/ PRÍNCIPE CABALLERO DEL ÓRDEN DE SANTIAGO/ASSIMISMO 24 PERPETVOS DESTA CIVDAD/SE ACABÓ/AÑO DE 1723".

A partir de 1724, por espacio de diez años Miguel de Quintana con la supervisión de Matías de Figueroa, "maestro de la obra de este Colegio", estuvo extrayendo piedras de diversas canteras, con destino a la construcción de la fachada y portada de este edificio, cuya edificación y volumen de documentos se escapan al espacio previsto en este artículo. Solo nos interesa destacar la portada del

\footnotetext{
49 AHUSe., libro 21, f. 573, libro 169, f. 252.

${ }^{50}$ Falcón, 1991: 38-39.
} 
vestíbulo, que da acceso al patio principal por el oeste. Fue construida en 1725 por Matías de Figueroa ${ }^{51}$ (Figura 5). Se estructura a base de pilastras cajeadas de orden compuesto. Sobre el dintel hay un frontón roto y enroscado, con dos diminutas figuras de león, como en los balcones centrales del patio. Se culmina con un frontón de triple inflexión que alberga una cabeza de querubín. Remata el conjunto tres tallas femeninas que deben aludir a virtudes. La de la izquierda que conserva su atributo, una columna, representa la Fortaleza. Esta decoración escultórica la atribuimos a Quintana.

El matrimonio integrado por Miguel de Quintana y María Gómez tuvo cuatro hijos: Luisa, María de la Concepción, Isabel y Ángel Francisco ${ }^{52}$. El único varón trabajó junto a su padre en San Telmo, donde figura en las nóminas de 1731 y 1732. En este último año consta al frente de los canteros, en calidad de oficial. Una de sus actividades fue la de medir las piedras procedentes de las canteras, con destino a la fachada y portada principal ${ }^{53}$.

\section{IGLESIA DE SANTA CATALINA}

A esta iglesia gótico-mudéjar, que ocupa el espacio de una antigua mezquita, se le agregó en la cabecera, lado del evangelio, la capilla sacramental, una de las joyas del barroco sevillano del siglo XVIII, al mismo tiempo que evidencia influencias de Borromini. Proyectada en 1721 por Leonardo de Figueroa, quien se ufana de este proyecto al afirmar que "será una de las primeras capillas que haya en la ciudad", su ejecución se dilató hasta 1736, seis años después del fallecimiento del arquitecto. Responde a la estética del barroco policromo tan característico de Leonardo. En ella intervino su equipo habitual, integrado por su hijo Matías, Pedro Duque Cornejo y Miguel de Quintana, entre otros. Fue Sancho Corbacho quien documentó que la intervención del maestro cantero se centró en la realización del zócalo de mármol rojo y negro de los muros de la capilla (1724-27), así como en la ejecución de la estatua de la $\mathrm{Fe}$ (1724) que corona la linterna, labrada en dos piedras blancas procedentes de una cantera de Antequera ${ }^{54}$ (Figura 6). Una para la talla de la imagen, y la otra para su basamento cilíndrico, ornamentado con motivos auriculares y apliques de azulejos azul cobalto, como el resto de la linterna. La

${ }^{51}$ AHUSe., libro 23, f. 565-566.

52 APSa., Padrones, libro 20 (1725-27) y libro 23 (1731-32).

${ }^{53}$ AHUSe., sección San Telmo, libro 91K, hijuela 133: "Nómina de 2 a 5 de enero de 1731". Percibía 8 rs. diarios. Libro 172 (año 1732), f. 36r.: "It. A Ángel de Quintana oficial de cantería se pagan 931 rs., 19 mrs. de vellón, por data de 21 de junio de 1732, por lo que importa la hijuela 209 de los jornales de canteros que va desde 16 hasta 21 del dicho mes de junio".

${ }^{54}$ Sancho Corbacho, 1952: 94-97. Morales, 1997: 11-18. 
Fe porta una cruz de hierro en la mano izquierda y en la derecha falta el cáliz. Sobre su cabeza hay una voluminosa corona imperial. En el curso de la restauración llevada a cabo en esta capilla en 1977, los restauradores Carmen Ortiz González y Ramón Romero Dorda realizaron la limpieza de la talla, que no presentaba ninguna patología. Únicamente restauraron o renovaron las piezas metálicas, tales como la cruz y la corona. La imagen luce una espléndida cabellera que cubre sus hombros, y viste túnica con lazo en la cintura y manto recogido ${ }^{55}$.

Fecha de recepción: 20 de octubre de 2020.

Fecha de aceptación: 2 de mayo de 2021.

\section{BIBLIOGRAFÍA}

Alonso Ruiz, Begoña (1991): El arte de la cantería: los maestros trasmeranos de la Junta del Volo. Santander: Universidad de Cantabria - Asamblea Regional de Cantabria

Barrio Loza, José A./Moya Valgañón, José G. (1981): “Los canteros vizcaínos (1500-1800)”. En: Diccionario biográfico. KOBIE, Boletín Espeleológico Vizcaino, 11, pp. 173-282.

Cajigas Aberasturi, Ana Isabel (2019): Canteros de Trasmiera. Historia social. Santander: Universidad de Cantabria.

Díaz Fernández, Ezequiel (2003): "Notas de cantería ostipense: Juan Antonio Blanco, cantero". En: Laboratorio de Arte, 16, pp. 507-521.

Falcón Márquez, Teodoro (1977): La capilla del sagrario de la catedral de Sevilla. Sevilla: Diputación provincial de Sevilla.

(1980): La catedral de Sevilla. Estudio arquitectónico. Sevilla: AyuntamientoDiputación provincial de Sevilla.

(1983): Iglesias de la Sierra de Cádiz. Cádiz: Caja de Ahorros Provincial.

(1991a): El Palacio de San Telmo. Sevilla: Ediciones Gever.

(1991b): "Jesucristo como modelo en el programa iconográfico del Palacio de San Telmo de Sevilla". En: Cuadernos de Arte e Iconografía, 7, pp. 256262.

(1992): "El patrimonio monumental y artístico del palacio de San Telmo". En: Isidorianum, 1, pp. 13-29.

(1997): El Palacio arzobispal de Sevilla. Córdoba: Cajasur.

55 Morales, 1997: 46. En el informe para la declaración en 1985 como Bien de Interés Cultural (BIC) de este monumento por la Junta de Andalucía, al maestro cantero se le denomina Miguel Quintero (en lugar de Quintana), asimismo se dice que la realización de la estatua de la Fe fue obra de Pablo Guizado, entallador que figura en las nóminas de esta capilla como el artífice de la decoración interior a base de yeserías. 
(2008/2009): "Influencias de los grabados de Dietterlin en la arquitectura sevillana". En: Laboratorio de Arte, 21, pp. 117-134.

(2011): "Pedro Romero, arquitecto del barroco sevillano (1638-1711)". En: Laboratorio de Arte, 23, pp. 225-251.

(2014): "La portada del palacio de San Telmo de Sevilla. Nuevas aportaciones e interpretaciones". En: Diálogos de Arte. Homenaje al profesor Domingo Sánchez-Mesa Martín. Granada: Universidad de Granada, pp. 277-290.

(2018): El Palacio arzobispal de Sevilla. Historia y patrimonio. Sevilla: Archidiócesis.

Gaeta, Letizia/García Luque, Manuel (2019): “Escultores italianos en España a comienzos del XVIII. Novedades sobre Domenico Lemico y Domenico Grasselli”. En: Archivo Español de Arte, 92, pp. 389-396.

Garmendia Arrubarrena, José (1986): Vascos en Cádiz. Siglos XVII-XVIII. San Sebastián: Fundación Kutxa.

Herrera García, Francisco J. (1988): "Lorenzo Fernández de Iglesias, un maestro cantero montañés en Andalucía occidental”. En: Atrio, 0, pp. 9-27.

(1990): Noticias de Arquitectura (1700-1720). Fuentes para la Historia del Arte Andaluz II. Sevila: Ediciones Guadalquivir.

Jos López, Mercedes (1986): La capilla de San Telmo. Sevilla: Diputación de Sevilla.

Mendioroz Lacambra, Ana María (1989): "Miguel de Quintana autor de la portada del Convento de la Merced, actual Museo de Bellas Artes de Sevilla”. En: Laboratorio de Arte, 2, pp. 261-266.

(1993): Noticias de Arquitectura (1721-1740). Fuentes para la Historia del Arte Andaluz VI. Sevilla: Ediciones Guadalquivir.

Morales, Alfredo J. (1997): "La capilla sacramental de Santa Catalina: un espacio barroco sevillano". En: Capilla sacramental de la iglesia de Santa Catalina. Madrid: Fundación Argentaria, pp. 19-36.

Pleguezuelo, Alfonso, 2004: "Proyecto de retablo para la capilla de la Virgen de la Antigua. Lienzo, H. 1734. Colección particular, Jerez de la Frontera”. En: Pleguezuelo, Alfonso/Valdivieso, Enrique (com.): Domingo Martínez. En la estela de Murillo. Sevilla: Fundación El Monte, pp. 210-211.

Quiles, Fernando (2007): Teatro de la gloria. El universo de la Catedral de Sevilla en el barroco. Sevilla: Diputación provincial-Universidad Pablo de Olavide.

Romero Torres, José Luis (2014): “Jerónimo Balbás, Pedro Duque Cornejo y otros artistas en el retablo mayor de la iglesia del Sagrario de Sevilla”. En: Sánchez-Mesa, Domingo/Sánchez-Mesa Martínez, Domingo/López-Guadalupe, Juan Jesús (coords.): Diálogos de Arte. Homenaje al profesor Domingo Sánchez-Mesa Martín. Granada: Universidad de Granada, pp. 259-277. 
Sancho Corbacho, Antonio (1952): Arquitectura barroca sevillana del siglo XVIII. Madrid: CSIC.

(1934): Arquitectura sevillana del siglo XVIII. Documentos para la Historia del Arte en Andalucía VII. Sevilla: Laboratorio de Arte. Universidad de Sevilla.

Sojo y Lomba, Fermín (1933): Los maestros canteros de Trasmiera. Madrid: Hulves y Cia. 


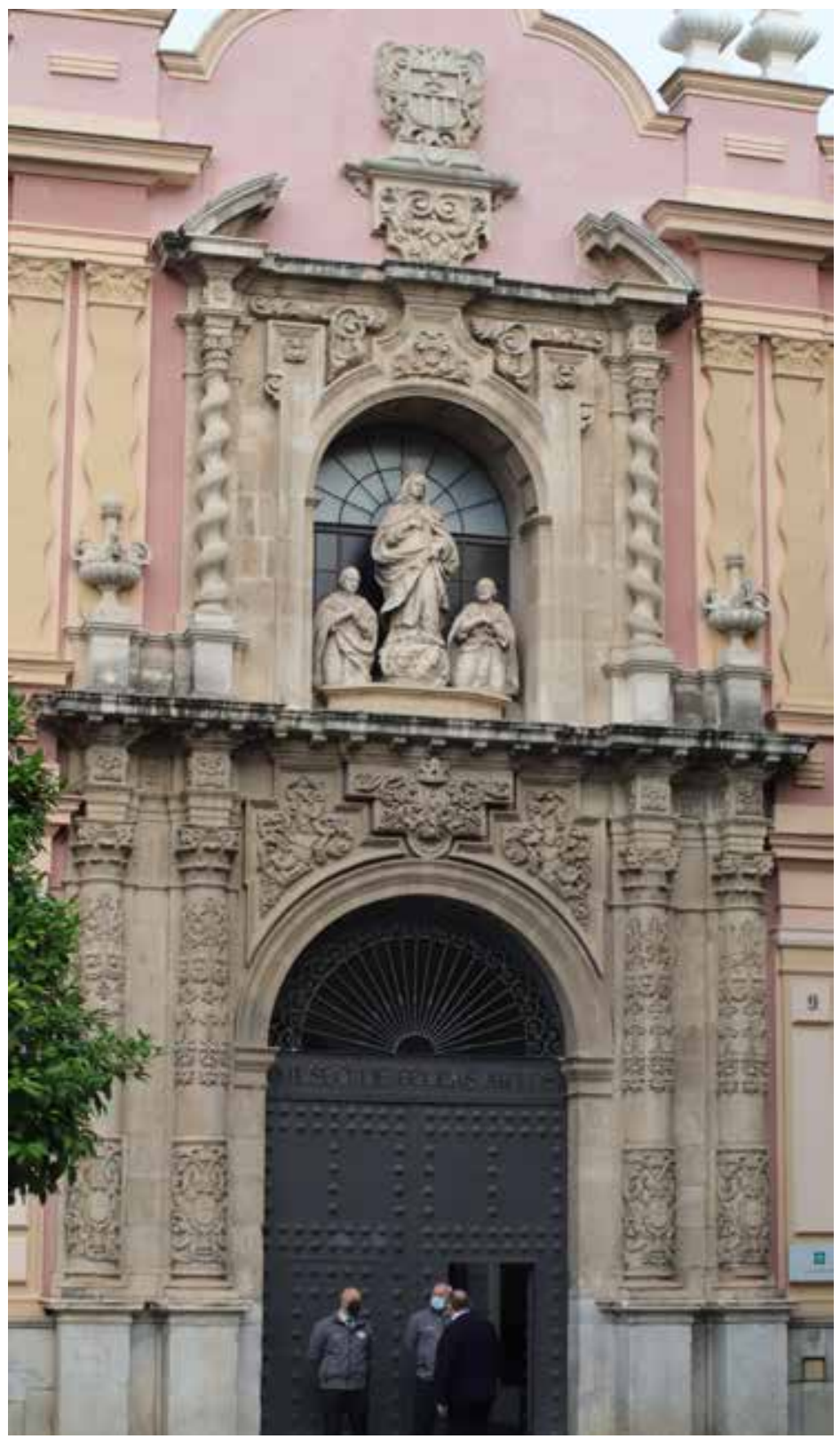

Figura 1. Miguel de Quintana, Portada principal de la iglesia del antiguo convento de la Merced, 1729, Museo de Bellas Artes de Sevilla. 


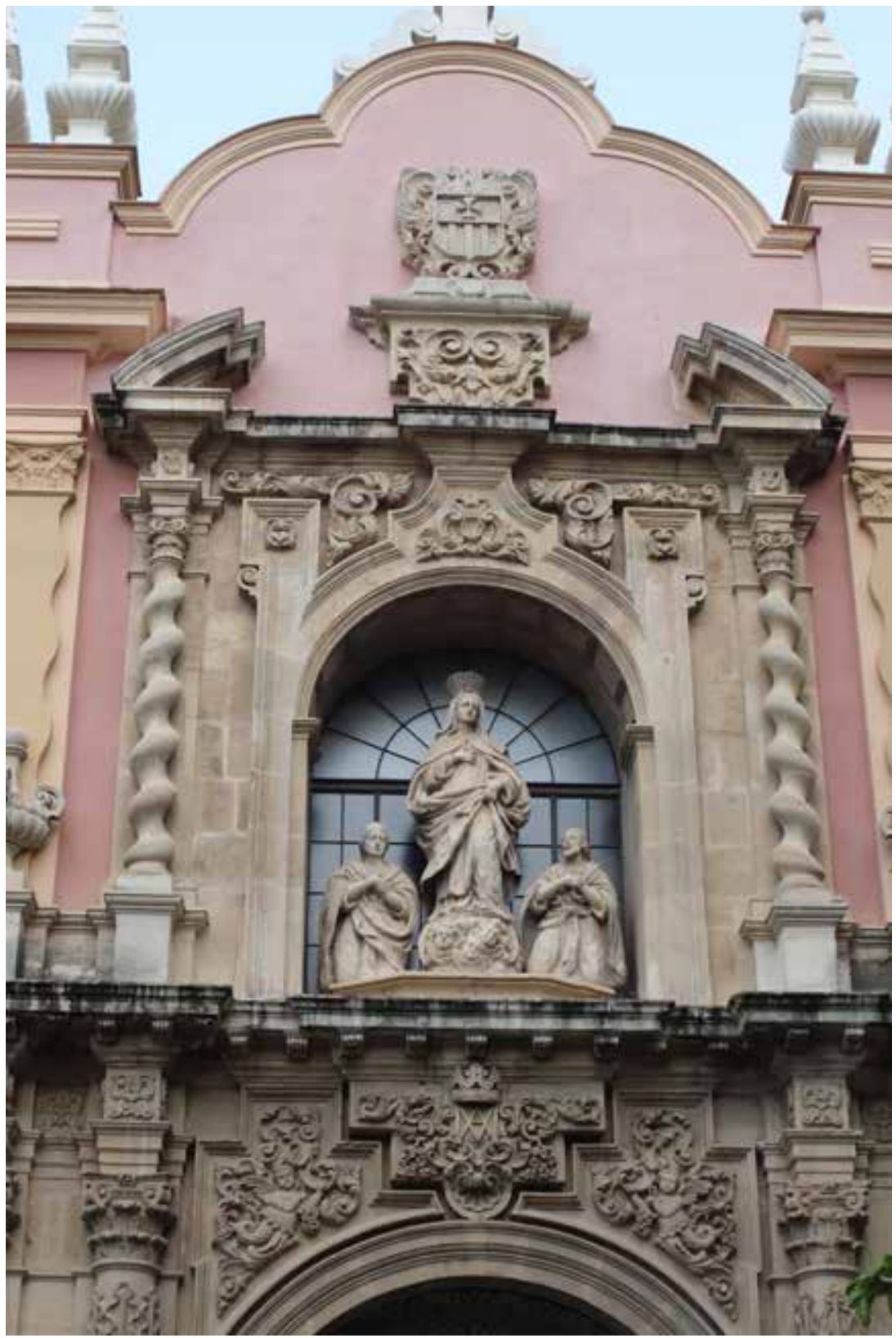

Figura 2. Miguel de Quintana. Segundo cuerpo de la portada principal de la iglesia del antiguo convento de la Merced, 1729, Museo de Bellas Artes de Sevilla. 


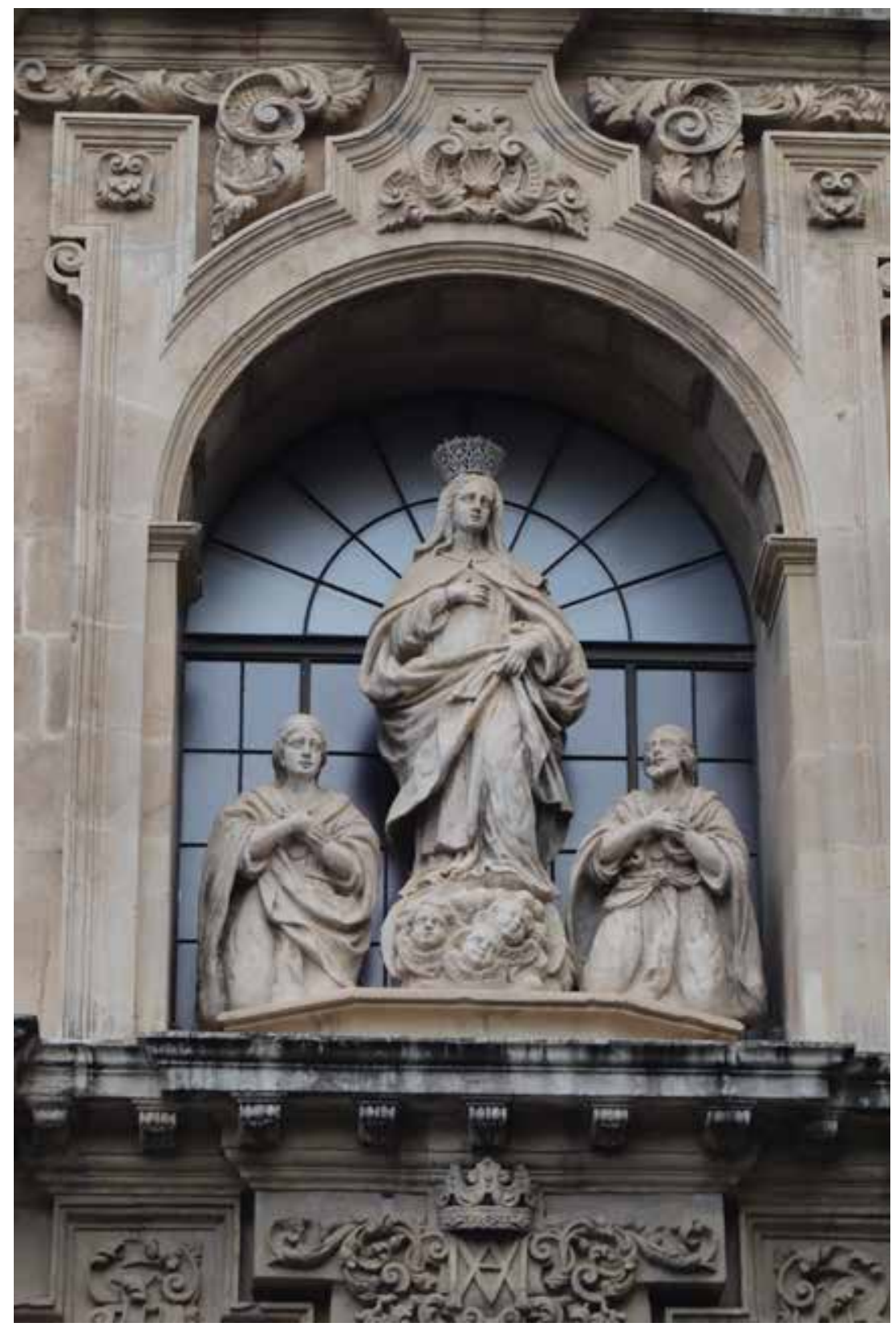

Figura 3. Miguel de Quintana. Grupo escultórico que preside la portada de la iglesia del antiguo convento de la Merced, 1729, Museo de Bellas Artes de Sevilla. 


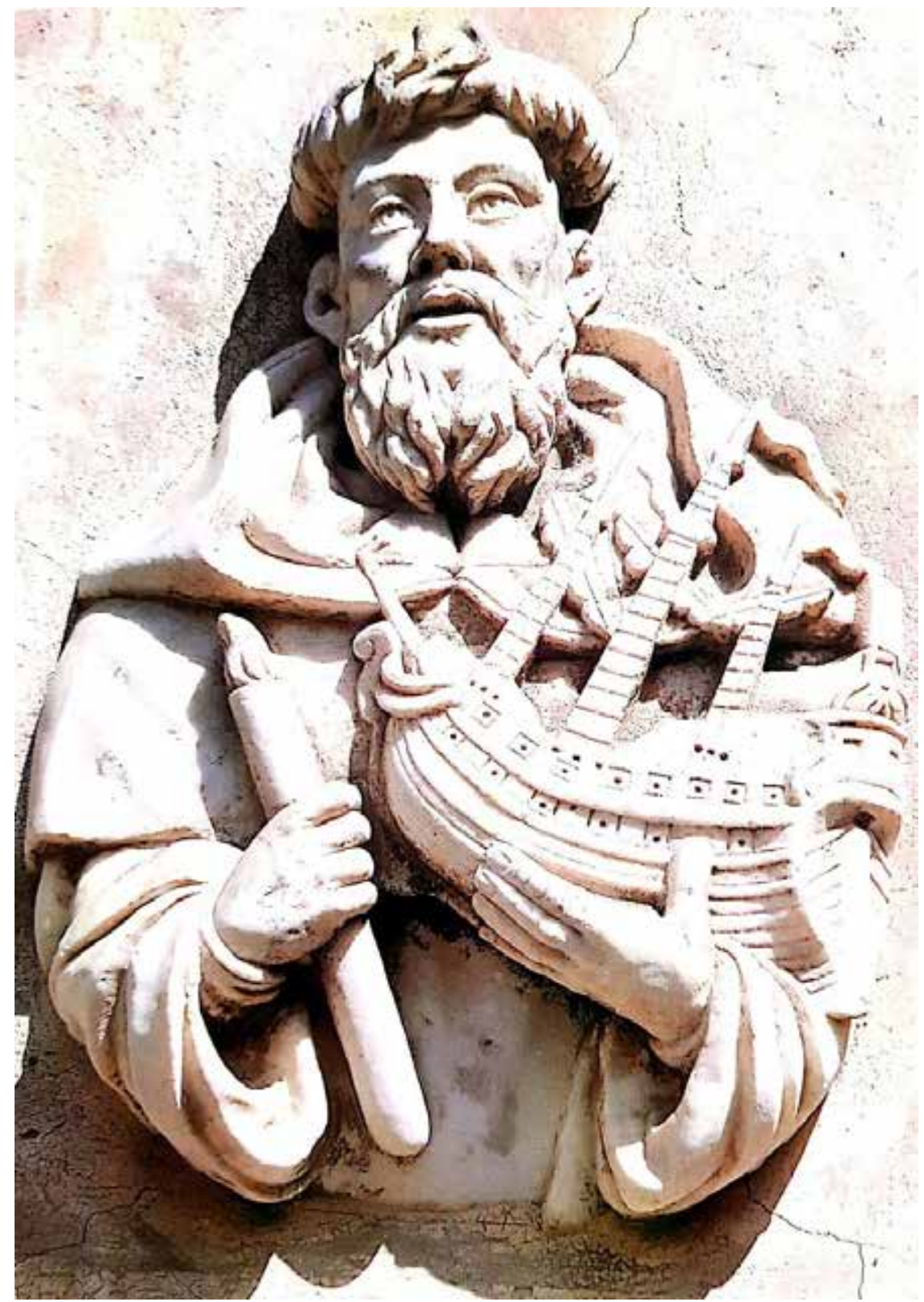

Figura 4. Miguel de Quintana, Relieve de San Telmo, 1723, palacio de San Telmo de Sevilla. 


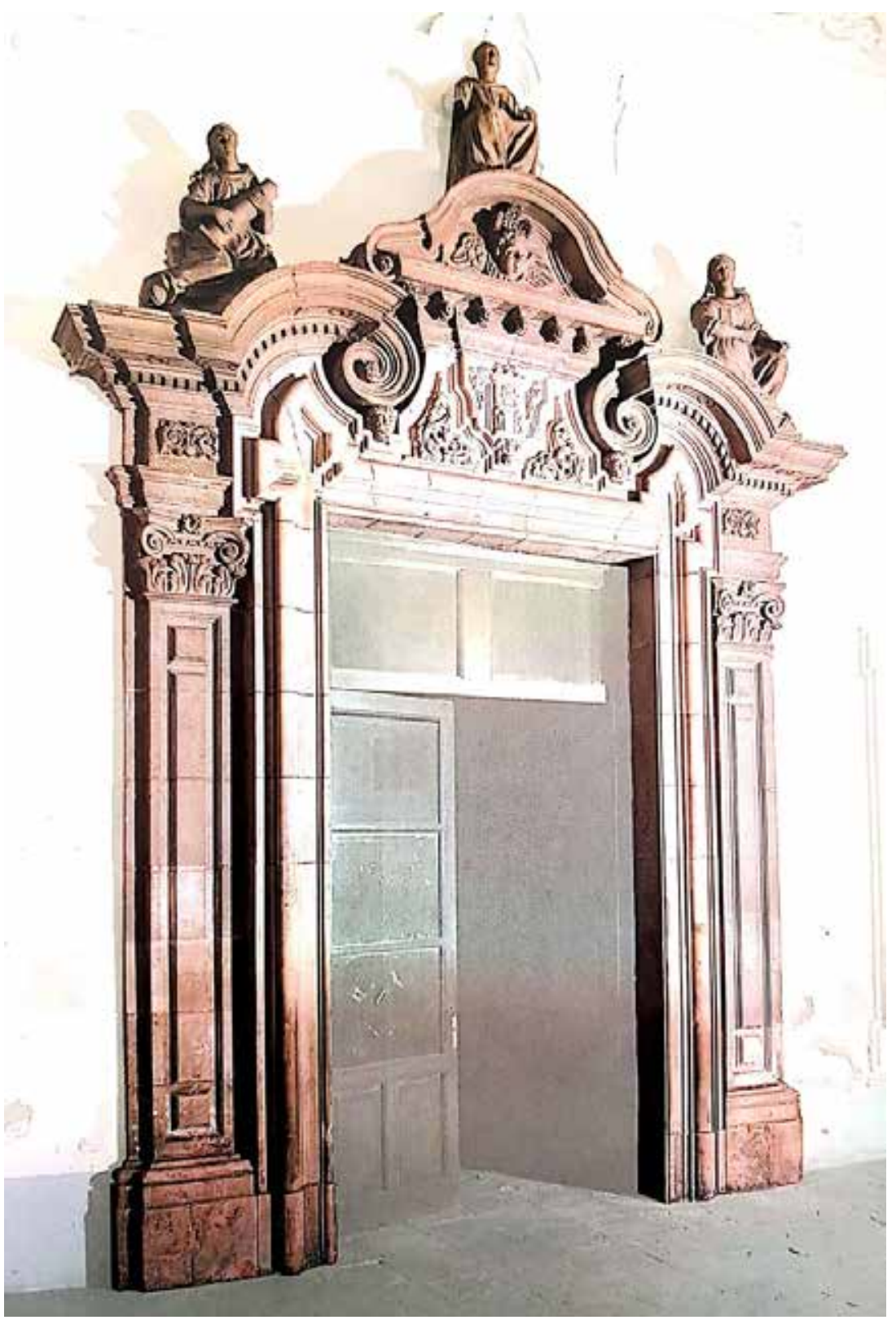

Figura 5. Miguel de Quintana, Portada del vestíbulo, 1725, palacio de San Telmo de Sevilla. 


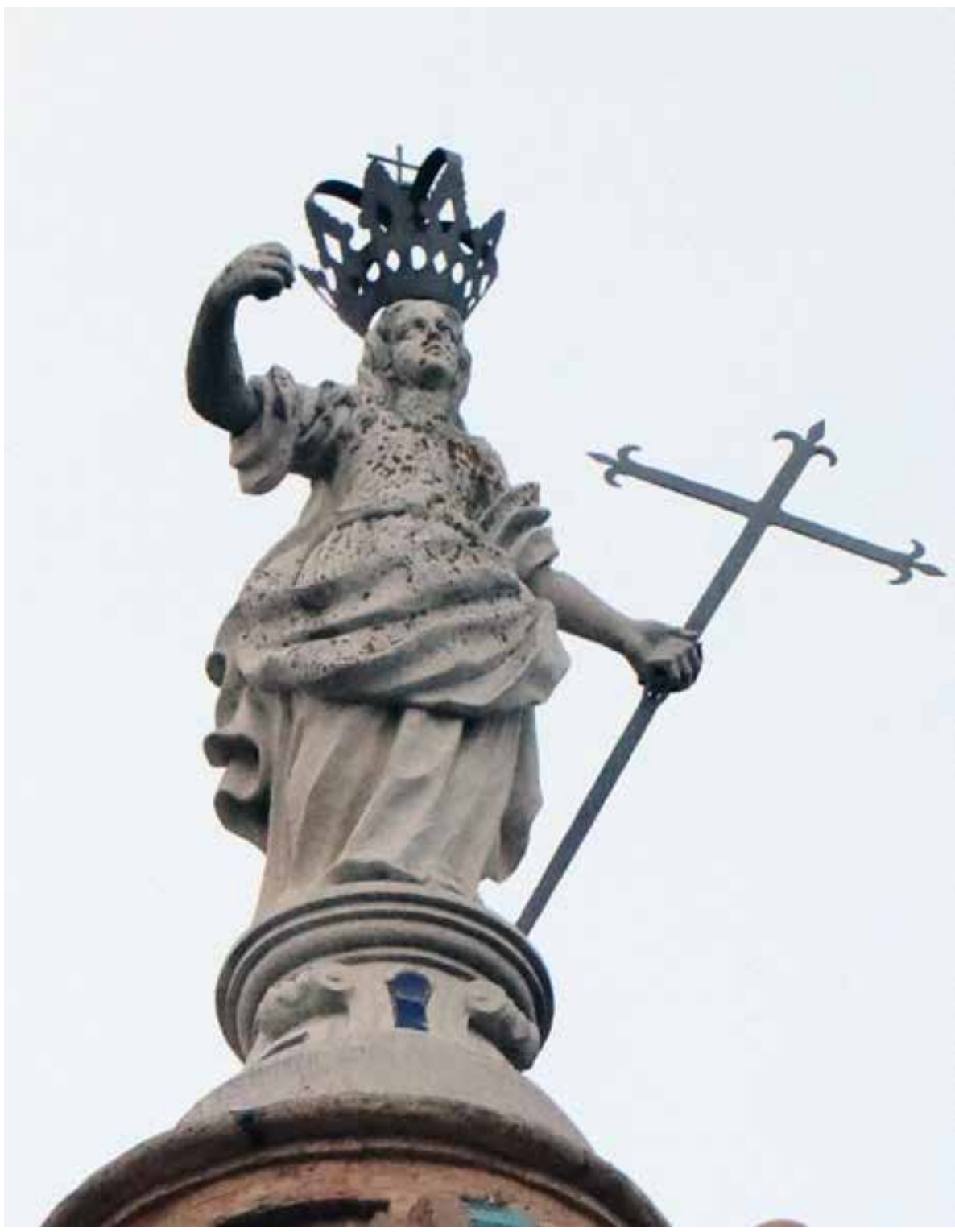

Figura 6. Miguel de Quintana, Estatua de la Fe, 1724, capilla sacramental de la parroquia de Santa Catalina de Sevilla. 